\title{
NOTAS
}

\section{LA REFERENCIA EN LA TRANSCRIPCIÓN (INFORMÁTICA) DE LOS TEXTOS ANTIGUOS. EJEMPLOS NEBRISENSES*}

RENÉ PELLEN

Universidad de Poitiers

\section{HACIA UN CAMBIO METODOLÓGICO: REFERENCIAR EL TEXTO CON RELACIÓN} AL LIBRO

Uno de los problemas fundamentales que se plantean a la hora de capturar los textos antiguos es el de la referencia con respecto a la fuente (manuscrita o impresa). Por lo común el transcriptor sólo considera el texto en sí mismo, haciendo caso omiso del marco en el que se presenta, o sea del libro (códice o incunable).

Esta práctica, que aísla el texto y lo trata globalmente como una cita externa, entraña muchas consecuencias negativas: desvanecimiento del concepto unitario del libro (incluso en el caso de una colección o una antología), supresión de toda relación con lo que antecede o sigue en el volumen, conversión del comienzo y el final relativos en comienzo y final absolutos, rechazo implícito de la cronología interna del libro, despreocupación por su estructura editorial y realización concreta.

Procede cambiar con urgencia este enfoque, devolviéndole a cada parte del libro el espacio que le corresponda en la representación informática, de modo que en los ficheros cada página, cada línea esté en su sitio, lo que supone un total respeto de las fuentes, incluso a veces en sus desvaríos o extrañezas. Y se aplicará este cambio lo mismo al libro que contiene un texto único que al que contiene textos múltiples.

A guisa de ejemplos, se examinarán a continuación tres libros de Nebrija que se publicaron en el último decenio del siglo XV: la Gramática castellana $(1492)^{1}$, el Lexicón $(1492)^{2}$ y el Vocabulario (c.1495) ${ }^{3}$.

* La Gramática, el Lexicón y el Vocabulario.

${ }^{1}$ Elio Antonio de Nebrija, Gramática castellana, Salamanca, [Juan de Porras], 1492 [BNM I2142], transcr. J. O’Neill, corr. S. B. Raulston, A. McCormick, vid. Admyte1, n. ${ }^{\circ} 6$ (n. 4). 
Las versiones informatizadas aparecieron en Admyte (1992) ${ }^{4}$, por otra parte, en el CD del Hispanic Seminary of Medieval Studies de Madison (1999) ${ }^{5}$. Las primeras siguen utilizadas en el sitio web de Admyte.com, lo mismo que en el CORDE de la Real Academia ${ }^{6}$ — salvo el Lexicón, que de momento no figura en este repertorio-.

\section{LA GRAMÁtICA DE NEBRIJA (1492)}

Haebler ${ }^{7}$ describe como sigue la edición de 1492: «68 hjs no fols - Sign: a$\mathrm{h}^{8} \mathrm{i}^{4}$ - a línea tirada. - 34 líneas en cada plana». Si se compara con la versión de Admyte, se hace patente la diferencia entre incunable y transcripción, siendo el 66v28 el último renglón del fichero. Lo mismo se observa en la edición de Esparza y Sarmiento (1992) ${ }^{8}$. Faltan, al parecer, dos folios. En realidad, el texto propiamente dicho ocupa los ff. a2r-i3v, como queda apuntado en PhiloBiblon $^{9}$ y puede comprobarse en los facsímiles que publicaron Galindo Romeo y Ortiz Muñoz (1946) ${ }^{10}$ o Esparza y Sarmiento, quedando en blanco el i4rv. El mismo impresor indica a pie de página que empieza el texto con el folio <a.ii $>$, ya que el primero es el de la portada, que en su recto trae el título de la obra ${ }^{11}$.

2 Elio Antonio de Nebrija, Dictionarium latino-hispanicum, Salamanca, [Juan de Porras], 1492 [BNM I-1778/1], transcr. A. Cortijo, vid. Admyte1, n. ${ }^{\circ} 4$ (n. 4). Ref. Lexicón.

3 Elio Antonio de Nebrija, Dictionarium hispano-latinum, Salamanca, [Juan de Porras], c.1495 [BNM I-1778/2], transcr. J. O’Neill, corr. A. Cortijo, vid. Admyte1, n. ${ }^{\circ} 5$ (n. 4). Ref. Vocabulario.

${ }^{4}$ ADMYTE [Archivo digital de manuscritos y textos españoles], 1993-1999, ed. por Francisco Marcos Marín, Charles B. Faulhaber, Ángel Gómez Moreno et al. Madrid, Micronet - Biblioteca Nacional - Sociedad Estatal Quinto Centenario, 3 CD-ROMs. Admyte0, Admyte1, Admyte II.

5 Electronic Texts and Concordances of the Madison Corpus of Early Spanish Manuscripts and Printings, prepared by John O'Neill (Electronic Texts on CD-ROM Series; 14), Madison New York, The Hispanic Seminary of Medieval Studies. Ref. CD-ROM Madison 1999.

${ }^{6}$ Real Academia Española, Corpus diacrónico del español, http://www.rae.es (2006-2008). Abrev. CORDE.

7 Konrad Haebler, Bibliografia Ibérica del siglo XV: enumeración de todos los libros impresos en España y Portugal hasta el año de 1500, Mansfield, Martino Publishing, 2000, 2 vols., vol. I, pág. 221. Reimpr. facsim. de la edición en 2 vols. public. en 1903-1917 por Martinus Nijhoff.

8 Elio Antonio de Nebrija, Gramática castellana, introd. y notas de Miguel Ángel Esparza y Ramón Sarmiento, Historiografía de la Lingüística Española, Clásicos Españoles, Madrid, SGEL Fundación Antonio de Nebrija, 1992.

9 PhiloBiblon, elab. por Charles B. Faulhaber y Ángel Gómez Moreno, Berkeley, University of California, 1999, CD-ROM; vid. sunsite.berkeley.edu/Philobiblon/phhm.htm.

10 Elio Antonio de Nebrija, Gramática de la lengua castellana, texto establecido sobre la ed. princeps de 1492 por Pascual Galindo Romeo y Luis Ortiz Muñoz, con una introd., notas y facsímil; pról. de José Ibáñez Martín, Madrid, Ed. de la Junta del Centenario, 2 vols., 1946.

11 Puede verse reproducción facsimilar en José Andrés Anguita, ed., Una aproximación a la «Gramática» de Nebrija, Sevilla, Alfar, 2005, pág. 31. 
Lo que pasa, pues, en todas las ediciones de la $G C$, tanto informatizadas como tradicionales, es que el transcriptor da por equiparables dos sistemas de referencia completamente distintos, sustituyendo $<$ a.ii $>$ por $<1$ r $>$ y así sucesivamente, sin preocuparse de la composición del libro por cuadernos.

La consecuencia más negativa de este método es que descarta la posibilidad de llevar a cabo ningún tipo de estudio relacionado con la estructuración en cuadernos.

Es imprescindible, por consiguiente, adaptar las referencias a la realidad del libro, de forma que empiece el texto propiamente dicho con la página $2 \mathrm{r}$ y termine con la $67 \mathrm{v}$, lo que significa una unidad más en el número de cada folio. El ejemplar que digitalizó Admyte es el I-2142 de la Biblioteca Nacional de España, en el que se suplió el folio 7 (según el sistema empleado) por el correspondiente del I-2651. Desde el nuevo enfoque, no se perdió el penúltimo folio del primer cuaderno sino el último (el $8^{\circ}$ ), lo que sucede con mayor frecuencia, siendo el primero y el último los folios más exteriores del cuaderno.

En cuanto al número de líneas en total y por página, conviene precisar las indicaciones de Haebler. Cada plana no tiene exactamente 34 líneas; se registran cuatro de sólo 33 (4r, 5r, 60v y 67v). Pero aun si se tiene en cuenta esta irregularidad, debería recogerse en las primeras 131 páginas de la versión informatizada un total de 4.450 líneas, cuando en realidad sólo reúne 4.433. Le faltan, pues, 17 líneas, que corresponden a las líneas en blanco que de vez en cuando introdujo el impresor. Una referenciación correcta de las líneas exige que se restablezcan escrupulosamente dichas líneas, para evitar que se convierta en página de 32 líneas una página regular de 34, como ocurre en la edición de Esparza y Sarmiento $(11 \mathrm{v})^{12}$.

Precisamente, como la $G C$ con frecuencia se cita por esta edición ${ }^{13}$, parece oportuno señalar en un cuadro la ubicación de las líneas en blanco y los cambios que sufre la numeración de las líneas cuando van reincorporadas al texto.

${ }^{12}$ Nótese que aquí se usa, como se hará en adelante, la foliación correcta (o corregida), que coincide con la descripción que arriba se proporciona del incunable.

${ }^{13}$ Sobre esta transcripción, vid. René Pellen, «Transcription des incunables, histoire de l'écriture et diachronie. Étude critique de l'édition du Ve Centenaire de la Gramática castellana par Esparza et Sarmiento (1992)», Bulletin Hispanique, 2006-1, págs. 67-222. 


\section{CUADRO 1}

«GC». Cambios en la numeración de líneas (respecto de la transcripción de Esparza y Sarmiento)

\begin{tabular}{|c|c|c|}
\hline Líneas en blanco & Ref. E.-S. & Ref. nueva \\
\hline $5 \mathrm{r} 3-4$ & $4 \mathrm{r} 3-31$ & $5 \mathrm{r} 5-33$ \\
\hline $9 \mathrm{v} 12$ & $8 \mathrm{v} 12-33$ & $9 \mathrm{v} 13-34$ \\
\hline $11 \mathrm{v} 11$ & $10 \mathrm{v} 11-12$ & $11 \mathrm{v} 12-13$ \\
\hline $11 \mathrm{v} 14$ & $10 \mathrm{v} 13$ & $11 \mathrm{v} 15-34$ \\
\hline $17 \mathrm{v} 27$ & $16 \mathrm{v} 27-33$ & $17 \mathrm{v} 28-34$ \\
\hline $27 \mathrm{v} 30$ & $26 \mathrm{v} 30-33$ & $26 \mathrm{v} 31-34$ \\
\hline $28 \mathrm{v} 31$ & $27 \mathrm{v} 31-33$ & $27 \mathrm{v} 32-34$ \\
\hline $42 \mathrm{v} 31$ & $41 \mathrm{v} 31-33$ & $42 \mathrm{v} 32-34$ \\
\hline $43 \mathrm{v} 24$ & $42 \mathrm{v} 24-33$ & $42 \mathrm{v} 25-34$ \\
\hline $44 \mathrm{v} 33$ & $43 \mathrm{v} 33$ & $44 \mathrm{v} 34$ \\
\hline $45 \mathrm{r} 31$ & $44 \mathrm{r} 31-33$ & $45 \mathrm{r} 32-34$ \\
\hline $46 \mathrm{r} 15$ & $45 \mathrm{r} 15-33$ & $46 \mathrm{r} 16-34$ \\
\hline $48 \mathrm{r} 3$ & $47 \mathrm{r} 3-33$ & $48 \mathrm{r} 4-34$ \\
\hline $65 \mathrm{r} 22$ & $64 \mathrm{r} 22-33$ & $65 \mathrm{r} 23-34$ \\
\hline $65 \mathrm{v} 11$ & $64 \mathrm{v} 11-33$ & $65 \mathrm{v} 12-34$ \\
\hline $66 \mathrm{r} 21$ & $65 \mathrm{r} 21-33$ & $66 \mathrm{r} 22-34$ \\
\hline $67 \mathrm{v} 23$ & $66 \mathrm{v} 23$ & $67 \mathrm{v} 24$ \\
\hline $67 \mathrm{v} 25-28$ & $66 \mathrm{v} 24-28$ & $67 \mathrm{v} 29-33$ \\
\hline
\end{tabular}

Estas líneas blancas no sólo añaden una dimensión particular a la disposición didáctica del texto, sino que representan, en un libro de esta categoría, breve y poco costoso ${ }^{14}$, un verdadero lujo, que ha de valorarse, además, como un esfuerzo notable por mejorar a un tiempo la legibilidad y la manejabilidad del tratado.

${ }^{14}$ Véase Víctor Infantes, «1492: una cultura entre el libro y el lector», Gramática y humanismo. Perspectivas del Renacimiento español, ed. por Pedro Ruiz Pérez, Madrid, Ediciones Libertarias - Ayuntamiento de Córdoba, 1993, págs. 57-85. 


\section{EL LEXICÓN (1492)}

El primer diccionario de Nebrija salió a luz el mismo año que la $G C$ y en las prensas del mismo impresor, Juan de Porras ${ }^{15}$. Conviene descartar, de primera entrada, los ejemplares que pertenecen a la edición falsificada, probablemente posterior a 1492, «pese a que en el colofón figure tal año» (Colón y Soberanas $\left.{ }^{16}\right)$. El incunable que se digitalizó en Admyte1 es el I-1778 de la Biblioteca Nacional, cuya colación, como la del facsímil que publicaron Colón y Soberanas (Biblioteca Universitaria de Barcelona, Inc. 481), es la siguiente:

$$
a^{8}, a^{12}, b^{10}, c^{8}, d^{10}, e^{10}, f^{10}, g^{8}, A^{8}-K^{8}, L^{6}
$$

Consta, pues, de 162 folios o 324 páginas. Ahora bien, cuando se coteja esta colación con la versión de Admyte (1993), salta a la vista que no coincide la descripción del incunable con la realidad del fichero, puesto que éste termina en el folio 158. Faltan cuatro folios, de los que dos corresponden a lagunas del incunable I-1778 que ha servido de base para la transcripción (F2 y F7) ${ }^{17}$. Estas lagunas originan dos disturbios mayores; en primer lugar, desaparecen del diccionario 4 páginas enteras («Phaestias» - «Phoebigena» y «Possessiuum» «Praemissio»); por otra parte, sigue de manera continua la foliación del texto, de modo que después de 115v2:48 «Phaestus» se encuentra la línea 116r1:1 «Phoche», y después de 119v2:48 «Possestrix», 120r1:1 «Praemoderor». Es de interés para los que consulten la base de datos Admyte.com subrayar que el Lexicón continúa (en 2008) con estas lagunas. Pero de todos modos, faltan otros dos folios, cuya ausencia no tiene explicación.

Otra versión posterior del texto (1999) apareció en el CD que publicó el Hispanic Seminary of Medieval Studies (en adelante $M a d)^{18}$. Esta transcripción se realizó a partir del incunable I-1256 de la Biblioteca Nacional y presenta el texto completo, sin lagunas en F2 y F7. El último folio, sin embargo, es el $161 v$, lo que de nuevo supone una disconformidad con la colación en la que

15 Sobre la identificación del «Impresor de la Gramática», vid. María Antonia Varona García, «Identificación de la primera imprenta anónima salmantina», Investigaciones Históricas, vol. 14, 1994, págs. 25-33.

16 Elio Antonio de Nebrija, Diccionario latino-español (Salamanca 1492), ed. facsímil, estudio preliminar por Germán Colón y Amadeu-J. Soberanas, Barcelona, Puvill, 1979, pág. 34.

17 Cabe enmendar la nota 11 de Gloria Guerrero Ramos, El Léxico en el «Diccionario» (1492) y en el «Vocabulario» (¿1495?) de Nebrija, pról. de Manuel Alvar Ezquerra, Sevilla, Universidad de Sevilla - Ayuntamiento de Lebrija, 1995, pág. 58: «El ejemplar catalogado con la signatura I/1778 (1) está completo».

18 Elio Antonio de Nebrija, Dictionarium latino-hispanicum, Salamanca, [Juan de Porras], 1492 [BNM I-1256], transcr. J. O’Neill, vid. CD-ROM Madison 1999 (n. 5). 
coinciden todas las descripciones bibliográficas, y, a las claras, una discrepancia (¿breve, extensa?) entre la realidad de los cuadernos y el texto que se inserta en cada uno de estos espacios.

Para resolver el problema es preciso examinar detenidamente la estructura del incunable ${ }^{19}$. Lo primero que se observa, si se compara la versión de Admyte $(A l)$ con el incunable $(I)$, es que la página $1 \mathrm{v}$ de $A l$ transcribe la página de signatura $<$ a.i $>$ de $I$, lo que supone una evidente contradicción, ya que la signatura tipográfica siempre aparece en el recto, no en el verso de un folio. Consecuentemente, el cuaderno $a$ («infinitiuo uerbi impersonalis») termina en $7 \mathrm{v}$. El texto que sigue, principio del diccionario («Lexicon hoc est...»), está encabezado con la referencia 8r, aunque esta página forma parte del cuaderno $b$, como viene indicado por la signatura $\left\langle\mathrm{a}^{\prime} . \mathrm{i}\right\rangle^{20}$.

La versión del HSMS (como es lógico) respeta el intervalo entre tasa y principio del Prólogo, que queda en blanco, incorporándolo en la referencia como 1v (sin texto), de suerte que empieza el Prólogo en 2r, presentación más acorde con el recto <a.i>. Toma en cuenta, asimismo, el verso del folio 8 , igualmente en blanco. Lo que pasa, en realidad, es que el impresor introdujo la primera signatura en la página en la que empezaba el texto, por considerarse la de la tasa página preliminar. Pero la materialidad del cuaderno no viene modificada por esta discrepancia entre texto y libro, terminando el Prólogo en $<a 7 r>$ del impresor, $8 \mathrm{r}$ del libro, referencia que conviene restituir para que se corresponda la foliación de la transcripción con el esquema del incunable.

En el segundo cuaderno $\left(a^{\prime}\right)$ es donde se concentran la mayoría de los problemas que provocan desórdenes en la foliación del Lexicón. Y hasta cierto punto se repite la misma discordancia que en el primero, puesto que, siendo un cuaderno de 12 folios, se acaba en 11v según la signatura tipográfica, que empieza con $<$ a.i $>$. Como no existe ninguna ruptura entre la página $11 \mathrm{v}$ y la $<\mathrm{b} . \mathrm{i}>$ del impresor («Apostata» / «Apostasis») y tampoco se nota laguna de ningún tipo en el desarrollo del texto, el origen del desfase hay que buscarlo en el inicio del cuaderno. Efectivamente, el hiato se sitúa entre el Prólogo y el comienzo del diccionario, o sea entre la página $8 \mathrm{r}(<\mathrm{a} 7 \mathrm{r}>$ según el impresor $)$ y la primera del Lexicón (<a.i $>$ ), a todas luces segunda del cuaderno contando a partir del final. Quedó, pues, en blanco, el folio a'1 al momento de planificar el cuaderno, aunque no se sabe «si estaba prevista su anulación en el momento de ofrecer los ejemplares» ${ }^{21}$.

${ }^{19}$ Quiero agradecer la ayuda que con tanta amabilidad y eficacia me prestaron, por un lado, Abraham Madroñal, entonces Subdirector del CORDE, y Rosa Arbolí, Bibliotecaria de la Real Academia, por otro Julián Martín Abad, Jefe del Servicio de Manuscritos, Incunables y Raros de la Biblioteca Nacional.

${ }^{20}$ Para evitar toda confusión entre los dos cuadernos iniciales, se denominará en adelante $<$ a $>$ al primero y $<$ a'> al segundo.

21 J. Martín Abad, 2006, carta particular. 
Si se restablece este folio a' 1 en la foliación, el cuaderno $a$ ' está ya completo $(1+11)$ y las referencias correctas del texto (desde «Lexicon» a «Apostata») son 10r-20v, quedando el 9rv en blanco, lo mismo que la página 8v. Así se restaura, no sólo la coherencia directa entre la foliación de cada página y la colocación del texto en el folio correspondiente, sino también la perfecta concordancia entre la foliación del texto en su totalidad y la realidad de los cuadernos.

Más que deseable, es imprescindible que en adelante las transcripciones del Lexicón se conformen con la repartición del libro en 19 cuadernos de distinta extensión: uno de 6 folios ( $L$ o último), 13 de 8 ( $a, c, g, A$ - $K$, respectivamente el primero, $\left.\operatorname{los} 4^{\circ}, 8^{\circ}, 9^{\circ}-18^{\circ}\right), 4$ de $10\left(b, d, e, f\right.$, respectivamente $\left.3^{\circ}, 5^{\circ}-7^{\circ}\right)$ y uno de 12 ( $a^{\prime}$ o segundo). Coincidirían de esta manera las referencias del texto, cuaderno por cuaderno, con los 162 folios del incunable.

Para resumirlo todo en un cuadro cómodo, he aquí las características de $A l$ y $A d m$ respecto de $I$.

CUADRO 2

Cotejo de las referencias en I, Al y Mad

\begin{tabular}{|c|c|c|}
\hline Incunable & Admyte & Mádison \\
\hline 1r tasa & 1r tasa & 1r tasa \\
\hline 1v blanco & 1v Prólogo & 1v blanco \\
\hline 2r Prólogo & & 2r Prólogo \\
\hline 8r fin Pról. & 7v-fin Pról. & 8r fin Pról \\
\hline 8v blanco & 8r Lexicón & 8v blanco \\
\hline 9 rv <a'1 $>$ blanco & & 9r Lexicón \\
\hline $10 \mathrm{r}<\mathrm{a}$ '2 $>$ Lexicón & & \\
\hline $21 \mathrm{r}<\mathrm{b} 1>$ & $19 \mathrm{r}$ & $20 \mathrm{r}$ \\
\hline $117 \mathrm{r}<\mathrm{F} 1>$ & $115 \mathrm{r}<\mathrm{F} 1>$ & $116 \mathrm{r}<\mathrm{F} 1>$ \\
\hline $124 \mathrm{r}<\mathrm{F} 8>$ & $120 \mathrm{r}$ & $123 \mathrm{r}$ \\
\hline $162 \mathrm{v}$ Fin & $158 \mathrm{v}$ & $161 \mathrm{v}$ \\
\hline
\end{tabular}

De lo cual se deducen las modificaciones que convendría realizar en las versiones existentes para conseguir una perfecta concordancia con $I$. A Al le faltan cuatro cuadernos porque tiene dos lagunas (F2 y F7) y, por otro lado, no toma en cuenta las páginas en blanco $(1 \mathrm{v}, 8 \mathrm{v}, 9 \mathrm{rv})$ de los primeros cuadernos. Mad se aleja de $I$ a partir de 9r, y como hace caso omiso del folio en blanco a'1, le falta un folio hasta el final. Para corregir las referencias inexactas, cabe añadir en $A l$ dos unidades a cada folio desde el 8r, y 4 desde el 120r; en Mád una unidad a cada folio desde el 9 r. 
Pero no es tarea tan fácil como parece, sencillamente porque varía el número de líneas por página, o incluso por columna en una misma página, cuando en la columna $a$ se agrega un artículo frente a la signatura tipográfica que ocupa la última línea en $b$ (v. g. 10ra tiene 49 líneas de texto, 10rb 48). Aunque lo habitual sea que la signatura ocupe la parte derecha de una línea totalmente en blanco, lo que se observa en 10r vuelve a producirse en 11r ó 14r; pero incluso en páginas exentas de signatura se encuentran columnas de 49 líneas (10va, 10vb, 11va, 11vb, 15ra, 15rb).

Esta variación no se menciona con suficiente claridad en las descripciones bibliográficas del Lexicón. En realidad el Lexicón tiene 47 columnas de 49 líneas y 12 de 47 . Ahora bien, puede que se trate de un aspecto significativo del texto y en modo alguno fortuito. Significativo, por lo menos, en cuanto a la fabricación del libro, puesto que sólo 4 cuadernos presentan columnas de 49 renglones (los $a$ ', $b, J$ y $L$ ), y dos con una especial frecuencia (el primero del diccionario, $a^{\prime}$, con 32 columnas de las 36 que lo componen, y el último $L$, con 12 del total de 24). Por el contrario, las columnas de 47 líneas se encuentran todas en el cuaderno $E$ (10 de 12 , menos dos en $a$, pág. $5 \mathrm{v})$. Pero también procede recalcar que la última página del Prólogo ofrece columnas de 34 líneas, y la 5 r columnas de 44, detalle éste nada intrascendente ya que señala una bipartición del Prólogo: después de una introducción general y más bien lexicológica, Nebrija aborda lo que él mismo considera «el negocio principal» (5r2:40) y son una serie de puntualizaciones doctrinales y técnicas cuyo objeto es explicar cómo va elaborado el diccionario. Se notará que esta segunda parte del Prólogo se presenta como el principio de un capítulo, con hueco de tres líneas en cada columna e inicial ausente, pero mayúscula en la letra que sigue («[P]Rincipio», «[P]Rimera mente») —el comienzo del diccionario propiamente dicho (10r) resulta más modesto, con la $<\mathrm{L}>$ de la palabra inicial ya escrita en la rúbrica («Lexicon»)-

Volviendo a la variación en la extensión de las columnas, podría pensarse que en el cuaderno $a$ ' se buscó primero una pauta para la disposición de la materia, que sería a dos columnas de 49 líneas, usando el último renglón de la columna $b$ para la signatura tipográfica, cuando tenía que aparecer. Se repite este esquema en los rectos 10r, 11r y 14r, y todos los versos (o los rectos después del quinto) tienen columnas de 49 líneas. Pero se da alguna que otra excepción: columnas iguales de 48 antes de la signatura (12r, 13r), que se convierten en regla a partir de la página 19r (a'10r). Habida cuenta de la repartición de esas columnas largas en el Lexicón, es muy probable que se vincule, bien con cambios de cajista, bien con la génesis estructural del libro.

Efectivamente, vienen a coincidir diversos tipos de información (alógrafos, abreviaturas, etc.), brindando por lo menos una explicación para el esquema paradójico del incunable. 
El impresor usó en los dos cuadernos iniciales de la misma signatura tipográfica y dejó en blanco el primer folio del segundo cuaderno. Este paralelismo no puede expresar sino una ruptura cronológica: dicho cuaderno será en realidad el primero que se compuso, quedando el primer folio en espera de lo que se suele llamar preliminares del libro (tasa, título), lo que confirmaría el que se reproduzca en el cuaderno uno actual la misma disposición que en el segundo (primer folio en blanco y signatura $<$ a.i $>$ en el segundo folio). Llama la atención el hecho de que casi todas las columnas del segundo cuaderno tengan 49 líneas, como si se tratara de un ensayo, en busca del mejor modelo para el resto del diccionario — que luego se abandonó a favor de la norma de 48 líneas hasta los últimos cuadernos-... Por otra parte, el segundo cuaderno es el único de 12 folios, tamaño que en el tercero se redujo a 10, y en el cuarto a 8. Continúa la vacilación en los tres siguientes $(d-f)$, que otra vez se despliegan en 10 folios, pero se establece la norma de 8 a partir de $g$. Norma ésta que se conserva en el primer cuaderno, donde sólo se usan columnas de 48 líneas, como máximo.

En resumidas cuentas, bien parece ser que en un principio el impresor sólo tuviera entre manos el diccionario escueto, sin prólogo, y empezara a componerlo desde la página 10r («Lexicon hoc est...»), dejando un folio en blanco para la tasa y dándole al segundo la signatura $<$ a.i $>$. Nebrija le hubiera entregado el Prólogo después de acabarse la composición del diccionario y entonces se agregó un cuaderno, con la misma disposición que el primero original (folio uno en blanco y signatura <a.i>, etc.), salvo que se siguió la norma dominante en el Lexicón de 48 líneas por columna. Por otro lado, se empleó otra caja (tal vez a consecuencia de un reciente reparto de los tipos al acabarse la impresión de algún libro) o el cajista utilizó de otra manera las variantes tipográficas de que disponía. Se observa, en particular, un empleo mucho más frecuente que en ningún otro cuaderno, de dos variantes grafemáticas, la $<\mathrm{d}>$ uncial y la $\langle\mathrm{s}\rangle$ estirada $^{22}$

Desde esta perspectiva, la colación exacta del Lexicón sería sencillamente

$$
a^{8}, a^{12}, b^{10}, c^{8}, d^{10}, e^{10}, f^{10}, g^{8}, A^{8}-K^{8}, L^{6}
$$

que expresaría la discrepancia entre orden lógico (discursivo) y orden cronológico (en la composición, y probablemente en la redacción del libro).

${ }^{22}$ A guisa de ejemplos: en el cuaderno $a$ se recogen 132 casos de $<$ d $>$ uncial (67) y <s> estirada (65), o sea unos 9,5 casos por página, mientras que en $c$ y en $g$ se recogen respectivamente 44 y 48 casos $(30<\mathrm{d}>$ y $14<\mathrm{s}>$ en $c, 29<\mathrm{d}>$ y $19<\mathrm{s}>$ en $g)$, o sea un promedio de casi 3 y exactamente 3 por página. 


\section{El VOCABULARIO (c.1495)}

El segundo diccionario de Nebrija plantea menos problemas que el primero. Consta, según los bibliógrafos unánimes, de 106 folios, que se organizan de una manera muy sencilla: $\mathrm{a}^{10}-\mathrm{b}-\mathrm{n}^{8}$.

Sin embargo, la versión digitalizada que publicó Admyte (a partir de BNM I-1778) termina en 104v y la del HSMS (Mad en adelante, realizada a partir de BNM I-1255) ${ }^{23}$ en $105 v$. En realidad, el último folio está en blanco, de modo que resulta estrictamente exacta la referencia de $\mathrm{Mad}$, faltándole un folio a la de Admyte. Como es probable que muchos investigadores seguirán usando la versión de Admyte, bien a partir del CD, bien a través de la base en línea, se indicarán concisamente las discrepancias que ofrece respecto del incunable (y Mad):

\section{CUADRO 3}

Diferencias entre Admyte y el incunable

\begin{tabular}{|c|c|c|}
\hline Incunable & Admyte & Diferencia \\
\hline $1 \mathrm{r}$ tasa & $1 \mathrm{r}$ tasa & 0 \\
\hline 2r Prólogo & $1 \mathrm{v}$ & $1 / 2 \mathrm{f}^{\circ}$ \\
\hline $5 \mathrm{r}$ Vocab. & $4 \mathrm{r}$ & $1 \mathrm{f}^{\circ}$ \\
\hline $105 \mathrm{v}$ fin Vocab. & $104 \mathrm{v}$ fin Vocab. & $1 \mathrm{f}^{\circ}$ \\
\hline
\end{tabular}

Es obvia la razón del progresivo desvío en las referencias de Admyte: no se tomaron en cuenta las páginas en blanco, que reprodujo escrupulosamente el facsímil publicado por la $\mathrm{RAE}^{24}$. Están en blanco $<\mathrm{a} 1 \mathrm{v}>$ (verso de la tasa) y $<\mathrm{a} 4 \mathrm{v}>$ (verso del final del Prólogo). En este caso, se incorporó el folio de la tasa en el primer cuaderno, empezando el texto del Prólogo en el segundo folio, con signatura $<$ a.ij $>25$.

Cabe insistir en la extensión del primer cuaderno (10 folios), el más largo, como ocurre en el Lexicón: todos los siguientes sólo tienen 8 folios. Tal vez se explique por la doble presencia en este cuaderno de preliminares (tasa) y un

23 Elio Antonio de Nebrija, Dictionarium hispano-latinum, Salamanca, [Juan de Porras], c.1495 [BNM I-1255], transcr. J. O’Neill, vid. CD-ROM Madison 1999 (n. 5).

24 Real Academia Española, Vocabulario español-latino por Elio Antonio de Nebrija (Salamanca ¿1495?), reprod. facsím., Madrid, 1951. Reimpr. Madrid, Arco/Libros, 1989.

25 En la $G C$ y el Lexicón, las signaturas se notan $<\mathrm{i}\rangle,<\mathrm{ii}\rangle$, $<\mathrm{iii}>$, <iiii $>$ (excepciones en el Lexicón: <e.iiij>, <F.iiij>); sin embargo, las abreviaturas del diccionario, menos $<\mathrm{i}>$, se escriben $<\mathrm{ij}>$, <iij $>$, <iiij $>$. Las signaturas y los artículos del Vocabulario suelen usar $<\mathrm{j}>,<\mathrm{ij}>,<\mathrm{iij}>$, $<$ iiij $>$; faltan $<$ c.j $>$ e $<$ i.j $>$. 
prólogo. Se notará, sin embargo, que el Prólogo, muy breve (a2-a4), corresponde a la primera parte del prólogo del Lexicón; lo único que recuerde a la segunda parte queda condensado en una nota final (4r10-4r33) sobre el alfabeto castellano. La falta de todo desarrollo técnico acaso se deba a que ya se hiciera anteriormente en el Lexicón ${ }^{26}$.

En lo que respecta a la extensión de las columnas, la norma es de 48 líneas por columna, y sólo se recogen 4 columnas de 47 líneas (16rb, 52ra-rb, 66rb) y 5 de 49 (55va-vb, 105ra-rb, 105va). Las últimas indican probablemente una precaución del cajista, al temer que no quedara suficiente espacio para el final del diccionario y el colofón (en la última página se olvidan los dos títulos que debían encabezar las letras $\langle\mathrm{x}\rangle \mathrm{y}\langle\mathrm{z}\rangle$ 《De incipientibus a...» ${ }^{27}$ ).

Esta regularidad formal permite verificar más cómodamente la exactitud de la transcripción. El texto base del incunable (en concordancia con el facsímil de la RAE y excluidas las líneas blancas menos las internas, y la mención de $4 \mathrm{v}$ sin texto) presenta una extensión de 19.852 líneas. La versión de Madison, si se restan las cuatro líneas que se repiten ${ }^{28}$, alcanza un total de 19.759. Vale decir que se olvidaron 93 líneas, 14 de ellas en blanco; o sea, finalmente, 79 líneas de texto. Desde luego, estas lagunas exigen una revisión muy cuidadosa de dicha versión ${ }^{29}$. Pero este aspecto de la transcripción se examinará en otros estudios.

\section{El ESTUdiO DE NEBRIJA: NUEVOS DERROTEROS}

Con la revisión de las referencias en función de la realidad efectiva de los incunables, huelga decir que los estudios nebrisenses necesitan de una rápida y profunda puesta al día. No sólo porque más vale un sistema de referencia que esté en conformidad con las características de las fuentes, sino porque el análisis de textos tan extensos como los dos diccionarios exige el uso constante de la informática, y más si se añade la $G C$ y progresivamente otros textos de Nebrija, hasta disponer algún día del corpus completo de sus obras. $G C$ y diccionarios

\footnotetext{
26 En ocasiones, sin embargo, vuelve a utilizar las mismas abreviaturas que en el Lexicón, sin haberlas mencionado en el Prólogo: «no[uum]» 7r1:40, 7r2:25, 8v1:19 (aplicado, al parecer, al equivalente latino), «po[eticum]» 8v2:47, 9r2:17 (idem), «b[arbarum]» 21r1:27, 35r1:1 (id.), «pr[iscum]»73r1:31, 73r2:1 (id.); pero no se encuentran «ra[rum]», «os[cum]».

27 Esta rúbrica metalingüística tarda en aparecer hasta la letra $\mathrm{D}$ (<e.ij>, 36r), pero después se repite sistemáticamente.

28 Se trata de 23v2:27, 52r2:33, 57r2:3 (que se anticipa en 57r1:43), 88v1:45.

29 Por supuesto, lo mismo requiere la letra del texto, de la que no se ocupa directamente este artículo. El incunable presenta muchísimas erratas de imprenta: «Alguarismo arte [de] contar», no «arte contar»10v1:17, «expecta|[t]io», no «expectalio», que por lo común se conservaron en la versión digitalizada aunque se corrigieron algunas; en otros casos, son errores de lectura o transcripción: «muestren», no «muesten» 2r1:32, «romam», no «roman» 3r2:15, «anfractum», no «anfractuz» $5 \mathrm{r} 1: 45 \ldots$ El signo $<\mid>$ representa la supresión de alguna letra errónea.
} 
reúnen ya unas 55.000 líneas de texto. Resulta imposible examinar semejante conjunto a un tiempo globalmente y con detalle sin disponer de bancos de datos $\mathrm{y}$, sobre todo, de la posibilidad de verificar, en cualquier momento, el contenido de las bases (número de unidades, naturaleza de los elementos, etc.) ${ }^{30}$. Esto supone, para que todos puedan orientarse en los textos y a su vez controlar los resultados de los demás investigadores, que utilicen el mismo sistema de referencia y representación.

Es de desear, por lo tanto, que se adopten cuanto antes unas normas como las que se proponen en este artículo. Para la $G C$, convendría respetar el folio 1 de la tasa y darle a cada página la numeración que le corresponde añadiendo a cada folio una unidad. En el Lexicón, se ha de modificar la versión de Madison aumentando en una unidad las referencias de los folios a partir del $9^{\circ} \mathrm{y}$ adaptando los primeros a las peculiaridades de los cuadernos $a^{\prime}-a$. La versión del Vocabulario que publicó el CD de 1999 es correcta; bastaría suprimir la referencia de los folios $4 \mathrm{v}$ y $8 \mathrm{v}$ en blanco, así como las líneas que se repiten, y restituir las líneas olvidadas o enmendar las corrompidas para obtener una estructura idéntica a la del incunable.

No será cosa fácil sustituir por otro el sistema que se usó durante años, creyendo además que era el bueno. Pero no cabe duda que los estudiosos también habían notado lagunas y deturpaciones, lo que debería ayudarles a aceptar la necesidad de una actualización general y minuciosa.

Por otro lado, entre las urgencias metodológicas y eurísticas actuales destaca la de reducir la distancia que subsiste entre especialistas del libro y especialistas del texto ${ }^{31}$. Unos y otros se interesan por distintos aspectos de la misma realidad básica, el texto-libro, cuya forma no se limita a una dimensión estrictamente bibliográfica, sino que da acceso a una gran diversidad de significaciones.

En lo que se refiere a la investigación propiamente lingüística, sólo puede llevarse a cabo de una manera satisfactoria si toma en cuenta esta realidad del libro en cuanto producto histórico ${ }^{32}$, y más si pretende favorecer un enfoque cuantitativo y globalizador ${ }^{33}$. Una condición previa para este tipo de estudio es una transcripción fidedigna de los textos, exenta de lagunas y de errores de referenciación.

30 Vid. René Pellen y Francis Tollis, La «Gramática castellana» d'Antonio de Nebrija: grammaire d'une langue, langue d'une grammaire, Limoges, Lambert-Lucas, 2 vols., en prensa.

31 Vid. René Pellen, «Le Livre et le texte dans la description des incunables. Un exemple: la Gramática castellana de Nebrija (Salamanque, 1492)», Gazette du Livre Médiéval, n. ${ }^{\circ}$ 46, 2005 , págs. 37-48.

32 Cfr. Julián Martín Abad, Los libros impresos antiguos, Valladolid, Universidad de Valladolid, Secretariado de Publicaciones e Intercambio Editorial, 2004, passim.

33 Vid. René Pellen, Las abreviaturas en la grafía de la «Gramática castellana» (1492). Entre el manuscrito y el libro impreso, (Yuso, 1), Lugo, Axac, 2004. 